NBER WORKING PAPER SERIES

TARGET ZONES AND

EXCHANGE RATE DYNAMICS

Pau1 R. Krugman

Working Paper No. 2481

NATIONAL BUREAU OF ECONOMIC RESEARCH

1050 Massachusetts Avenue

Cambridge, MA 02138

January 1988

The research reported here is part of the NBER's research program in International Studies. Any opinions expressed are those of the author and not those of the National Bureau of Economic Research. 
NBER Working Paper \#2481

January 1988

\title{
Target Zones and Exchange Rate Dynamics
}

\begin{abstract}
This paper develops a highly simplified model of exchange rate behavior within the band under a target zone regime. It shows that the expectation that authorities will defend the band exerts a stabilizing effect on exchange rate behavior within the bard, even when the authorities are not actively intervening. The extent of stabilization can be related in a straightforward way to three factors: the sensitivity of the current exchange rate to expected depreciation, the volatility of the process driving exchange rate "fundamentals", and the credibility of the commitment by authorities to defend the target zone.
\end{abstract}

Paul R. Krugman

NBER

1050 Massachusetts Avenue

Cambridge, MA 02138 
It seems fairly likely that over the next few years the world monetary system will shift from one of freely floating rates to one in which governments commit themselves to keep exchange rates. within broad target zones. Despite the poprlarity of the concept, rowever, little researoh has been done on how such target. zones would operate in practice. In particular, how will exchange rates behave inside the target zone? A naive view might bo that the exchange rate will behave as if it were freely floating until it hits the edge of the band, then act like a fixed rate. With forward-looking sperulators, however, this cannot, be right: the expectation that the rate will be stabilized if it moves too far should affect its behavior ever when no active stabilization is taking place.

This paper presents a simple model of exchange rate behavior under a target zone regime. It, is essentially the same as the model presented in Krugman 419872 ; the differences are technical and expositional, but hoperully this version does produce some value-added. The main new elements are a cortinuous-time formulation of the process driving "fundamentals", ard a different. approach to the determination of the point at which the excharge rate reaches the edge of the band. In the present version of the model, it. is possible to derive a surprisingly simple closed-form analysis of the effects of a target zone on the exchange rate. As the paper shows, a target zone will lead to more stable excharige rate behavior within the zone than free floating. The extent of stabilization can be related in a straightforward way to the 
volatility of the fundamentals driving the excharge rate, the sensitivity of the exchange rate to expected appreciation or depreciation, and the credibility of the government commitment. to defend the target zone.

\section{THE MODEL.}

The exchange rate is assumed to be determired by two factors: "fundamentals", which evolve exogenously over time, and its owr expected rate of change. For the purposes of the model, it is not important to specify exactly what the fundamentals are; they might be monetary variables, or they might include a variety of other factors. All that we need is the assumption that the fundamental level of the logarithm of the exchange rate, which is the rate that would prevail if neither appreciation nor depreciation were expected, follows a diffusion process over time:

(1) $d x=0 \mathrm{~d} z$

which implies that the variarce over a period $t$ is simply o $e$.

It is useful to make a distinction between the exchange rate that would prevail in the abserce of active government. intervention, and the actual exchange rate that prevails given that intervention. Let us demote the $\log$ of the non-intervertion exchange rate as $\hat{s}$; it is related to the fundamentals and to expected charges in the exchange rates by 
$\cos \hat{s}=x+\gamma E[d s / d t]$

The actual exchange rate is assumed to be 1 imited by the commitment of the authorities to keep the rate withir a target. zone whose upper $l i m i t$ is $\ddot{s}$ and whose lower limit is $s$. Exactly how the authorities do this will not be specified in the model. One example that may clarify the point is to imagine that $x$ represents domestic credit, and that unsterilized intervention takes place when the exchange rate crosses the edges of the band. However, the model is more general than this, and may encompass a variety of different mechanisms. However it is done, we assume that whenever the shadow exchange rate would lie outside the target zone, the authorities are able to insure that the actual exchange rate remains at the boundary:

$$
\text { (3) } \begin{aligned}
s & =\bar{s} \text { if } \hat{s}>\bar{s} \\
s & =\hat{s} \text { if } \bar{s}>\hat{s}>s \\
s & =\underline{s} \text { if } s>\hat{s}
\end{aligned}
$$

Finally, for convenience it is helpful to choose units 50 that the band is centered around zero, i.e., $s=-\bar{s}$.

This is an extremely simple, rudimentary model of the exchange rate. In particular, two of the usual sources of interesting dynamics in exchange rate models are lacking: because of the random walk assumption about fundamentals, there is no room 
for responses to anticipated future shocks; also, the model lacks any "intrinsic" dynamics arising from slowly adjusting variables such as the distribution of world wealth or a sticky domestic price level. The payof to excluding these important and realistic issues it that it allows us to focus solely on the impact of the target zone on exchange rate dynamics; as we will see, we can derive some surprisingly clear and powerful conclusions about this i mpact.

EXCHANGE RATE BEHAVIOR INSIDE. THE BAND

Inside the target zone the actual and shadow exchange rates coincide, so that the exchange rate obeys the equation

$(4) s=x+\gamma E[d s / d t]$

The way to solve for exchange rate behavior is to look for an equilibrium in state space ... that is, for an equilibrium furction $s=g(x)$ such that $(4)$ is always satisfied. We note that when $x$ follows the diffusion process $(1)$, and where g(.) has a continuous second derivative, the expected rate of change of $s$ is determined by

(5) $E[d s / d t]=g^{\prime}(x) E[d x / d t]+(1 / 2) g^{\prime} \cdot(x) E\left[(d x / d t)^{2}\right]$

Since $x$ follows a diffusion process without drift, E[dx/dt $]=$ 
O; thus (5) reduces to

(E) $E[d s, d t]=g^{\prime} \cdot(x) \alpha^{2}$

It follows that the function g6.2 must be one for which

$(7) g(x)=x+c \cos 2,29 \cdot 6 x$

A function for which ( 7$)$ holds is

$(8) s=g(x)=x+A e^{r x}+E e^{-r y}$

where the value of $r$ may be determirued by substituting into $c$ ?:

$\cos x+A e^{r x}+B e^{-r x}=x+C \gamma d r e d e c A e^{r x}+B e^{-r x}$

i. mplying

$\cos r=\sqrt{2 / \gamma \sigma^{2}}$

The function (8) may be simplified by exploiting the symmetry of the assumed target zone. Since the target zone is centered around zero, and since there is ro drift in the furdanentals, we $\operatorname{can}$ assume that $g(0)=0$. This can orily be true if $B=-A$. So the function $\mathrm{g}(\infty)$ actually takes the form 
(11) $s=x+A\left[e^{r x}-e^{-r x}\right]$

As we will see in a moment, in a target zone A must be negative. This implies an S-shaped curve like that shown in Figure 1 , one for which $s<x$ in the upper part of the target zone and $s$ $>x$ in the lower part -.. that is, the $S$ lies below the 45 degree line in the upper part of the band and below it in the lower part. To understand this geometry, it is useful to rearrange $(7)$ to get

(12) s - $x=\left(r \alpha^{2}, 2\right) g^{\prime}(x)$

This says that the deviation of the exchange rate from the fundamental is proportional to the convexity of the fundamentals-exthange rate relationship. The reason is Iensen's inequality: since the expected charge ir $x$ is zero, the expected change in the exchange rate, which is a function of $x$, is positive where this function is convex, negative where this function is concave. In the lower part of the target zone, where gin is convex. the expected change in the exchange rate is positive; this "drags" the exchange rate ahove the 45 degree line. Conversely, ir the upper part of the zone the concavity of gro implies an expected depreciation that drags the exchange rate below the fundamental.

THE STABILIZING EFFECT OF THE TAFGET ZONE 
For the $g(x)$ drawn in Figure 1 , it is apparent that the target zone has a stabilizing ef fect on the exchange rate withir the band. The S-curve is al ways flatter than the 45-degree line, implying that fluctuations in the fundamental $x$ are less than fully reflected in the exchange rate; this is in contrast to free floating, in which the exchange rate would simply move up and down the 45-degree line. Interestirgly, this stabilization takes place even when the authorities are not actively defending the band: they only have to act when the fundamentals reach the levels $\bar{x}$ or $\underline{x}$. The reason is of course the expectation that they will defend the zone in future if necessary. Because of this, when the exchange rate is near the top of the band, it essentially has more room to go down than up, and conversely at the bot.tom; the market recognizes this, and this realization creates regressive expectations that stabilize the rate.

But does the function $g(x)$ actually lie in the position indicated? To show this, we need to demorstrate that A in equation (11) is in fact negative -- or what is the same thing, that $g(x)$ is in fact S-shaped.

The key to demonstrating this is to focus on how $\mathrm{g}(\mathrm{x})$ intersects the edge of the band. As drawn in Figure 1, the function $g(x)$ is exactly tangent to the limits of the target zone. What we need to show is that this is the correct depiction.

Figure 2 shows three possible ways in which $g(x)$ might intersect $\bar{s}$. In case 1 a convex $g c \infty$ crosses $\bar{s}$; in case 2 a 
concave $g(x)$ does the same thing; in case 3 a concave $g(x)$ is just tangent to $\vec{s}$. Which of these is the correct picture?

Recall two points. First, for $x>\bar{x}$, the level at which intervention by the authorities becomes necessary, we have $s=\bar{s}$. Thus in each case the actual exchange rate path is indicated by the heavy line, and the continuation of $\mathrm{g}(x)$ is indicated by the broken line. Secondly, intervention takes place only when $\hat{s}>\bar{s}$.

In each of the case indicated in Figure 2 . we denote by $E$ the first point at which the authorities intervene. What we can show is that in cases 1 and $2, \hat{s}$ at point $E$ is less than $\bar{s}$. That is, if the perceived fundamentals-exchange rate realtionship were as illustrated, the exchange rate at $E$ would be dragged back inside the band, contradicting the assumption that interverition becomes necessary at $E$. Only in case 3 is $\hat{s}>\vec{s}$. Thus only a tangency equilibrium is possible.

Consider what happers in cases 1 and 2 . If $s$ were to remain equal to $\mathrm{g}(x)$, then $\hat{s}$ at $\bar{x}$ would equal $\mathrm{g}(x)$ : that is the definition of $g(x)$. However in both cases 1 and 2 , if $x$ rises above $\bar{x}_{,} s i s$ constrained to 1 ie below $g(x)$; while if $x$ lies below $\bar{x}$, $s$ is still on $g(x)$. Thus the expected change in $s$ is unambiguously smaller than on $g(x)$. This implies that $\hat{s}<g(x)$ at E - contradicting the assumption that E lies on the band.

In case 3 , by contrast, at $x>\bar{x}$ we have $s>g(x)$, because $g(x)$ has turned downward. Thus in that case $\hat{s}>\mathrm{g}(x)=\bar{s}$, and the exchange rate does 1 ie on the band.

An alternative way of thinking about this result, which 
recalls our interpretation of the geometry of gexo, is to rote that in cases 1 and 2 the actual relationship between $x$ ard $s$ is less convex than g(x), implying an $\hat{s}$ below $g(x)$; only in case 3 is the relationship more convex.

A similar argument rules out any gex that tirns dowriward before reaching $\bar{s}$ : it is straight forward to show that the point at which such a $g(x)$ would cross $s$ or the way down is equally ursustainable.

The equilibrium g(x), then, must be precisely tangent to the top of the band cand by symmetry to the bottom as well. Th. This obsrvation allows us to derive a simple measure of the extent to which the target zone stabilizes expectations.

The key is that $g(x)$ reaches a maximum of $\bar{s}$ at $\ddot{x}$. Thus we may differentiate (11) to get

(13) $\partial s /\left.\partial x\right|_{\bar{x}}=1+\operatorname{rA}\left[e^{r \bar{x}}-e^{-r \bar{x}}\right]=0$

i mplying

(14) Al $\left.e^{r \bar{x}}-e^{-r \bar{x}}\right]=-1 / r$

At the same time,

$(15) \bar{s}=\bar{x}+A\left[e^{r \bar{x}}-e^{-r \bar{x}}\right]$

Thus we have 
(16) $\bar{x}-\bar{s}=1 / r=\left(\gamma / \sigma^{2} / 2\right)^{1 / 2}$

Now rotice that $\bar{x}-\bar{s}$ is a measure of the stabilizing effect of the band on the exchange rate. If there were no target zone, the exchange rate would fluctuate up and down the 45-degree line, and would therefore cross the value $\bar{s}$ wher $x$ equalled $\bar{s}$. What the target zone does is to create stabililizing expectations that keep the rate within the band for a widened range of fundamentals ewen when the authorities are not currently intervening; the extent of that widering is measured by $\bar{x}-\bar{s}$. We note that the stabilizing effect depends on two parameters: $\gamma$, which is the sensitivity of the exchange rate to the expected rate of depreciation, and $g^{2}$, the volatility of the fundamentals.

DYNAMICS WI TH IMPERFEGT CREDIBILITY

Up to this point we have assumed that the market believes that the authorities will in fact do whatever is necessary to deferd the target zone. Fealistically, of course, we need to take into account the possibility of a tailure to honor commitments. Fortunately, it is now straightforward to consjder the possibility of an imperfectly credible target zone.

Suppose that the market believes that the zone will be defended with probability $\phi$, and that with probability $1-\phi$ no 
response will occur when the exchange rate reachses the edge of the band. Then what will happen when the exchange rate does reach the edge of the band is a testing of the authorities' resolve. If the authorities do defend the band, they will now have a fully credible target zone; if they do not, the system will revert to free floating.

The consequence of such a test is shown in Figure 3 . Suppose that the exchange rate reaches the edge of the zone when the fundamentals reach the level $\tilde{x}$. Then if the authorities turn out to be credible, the exchange rate will move onto its full credibility schedule at $\mathrm{g}(\tilde{x})$, while if they do not it will jump to the 45-degree 1 ine at $\tilde{x}$. Since the market knows that one of these two events will occur, and since any expected jump would yield an infinite expected rate of appreciation, $\tilde{x}$ is defined implicitly by the requirement that the expected jump equal zero:

$(17) \bar{s}=\phi g(\tilde{x})+(1-\phi) \tilde{x}$

Corresponding to this new intersection is a fundamentals exchange-rate relationship defined by choosing $A$ so that the function intersects the edge of the band at $\tilde{x}$. We can call this $\tilde{g}(x, \phi)$. It is immediately apparent that $\tilde{x}<\bar{x}$, and therefore $\tilde{g}(x, \phi)$ is steeper than $g(x)$. Thus imperfect credibility reduces the stabilizing effect of the target zone. It is also apparent that as $\phi$ goes to one, $\dddot{g}(x, \phi)$ converges to $g(x)$, while as $\phi$ goes to zero it merges with the 45 -degree line. 
CONCLUSI ONS

This paper has set out a highly simplified model of exchange rate behavior under a target zone regime. The method of solution is one that is still somewhat unfamiliar in international economics, and the geometry -- in which the convexity of the relationship between fundamentals and prices plays a crucial role - is new and somewhat surprising.

The main substantive result is that the expectation that the authorities will defend a target zone will exert a stabilizing influence on exchange rate behavior inside the zone. The extent of this stabilizing influence depends on the sensitivity of the current exchange rate to exchange rate expectations, the volatility of the underlying determinants of the exchange rate, and the credibility of the authorities' commitment.

The obvious next step in this research is to make the model more realistic and interesting; this will involve both allowing a more complex process generating fundamentals, and introducing some "intrinsic" dynamics arising from such sources as sluggish price adjustmert. 


\section{REFERENCES}

Flood, Robert ard Garber, Peter (1983), "A model of stochastic process switchirg", Econometrica $51,537-551$.

Krugmar, Paul (1987), "Trigger strategies and price dynamics ir equity and foreign exchange markets". NBER working Paper $\$ 2759$

Mussa, Michael (1979), "Empirical regularities in the behavior of exchange rates and theories of the foreign exchange market.", in Policies for employmert, prices, and exchange rates, Carnegie Rochester Conference Series on Public Policy, 11. 


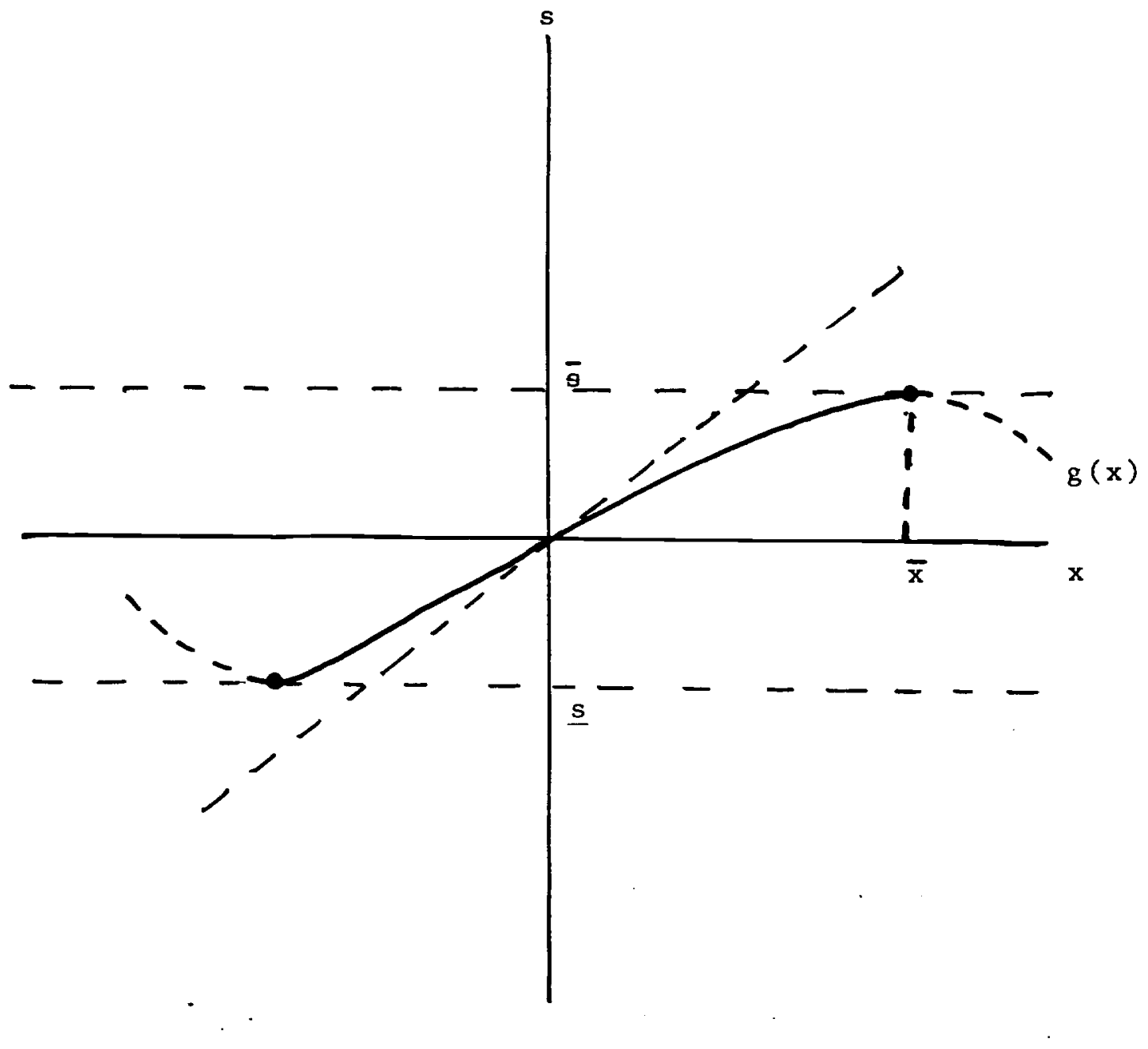

Figure 1: The fundamentals-exchange rate relationship 


$$
\text { the }
$$




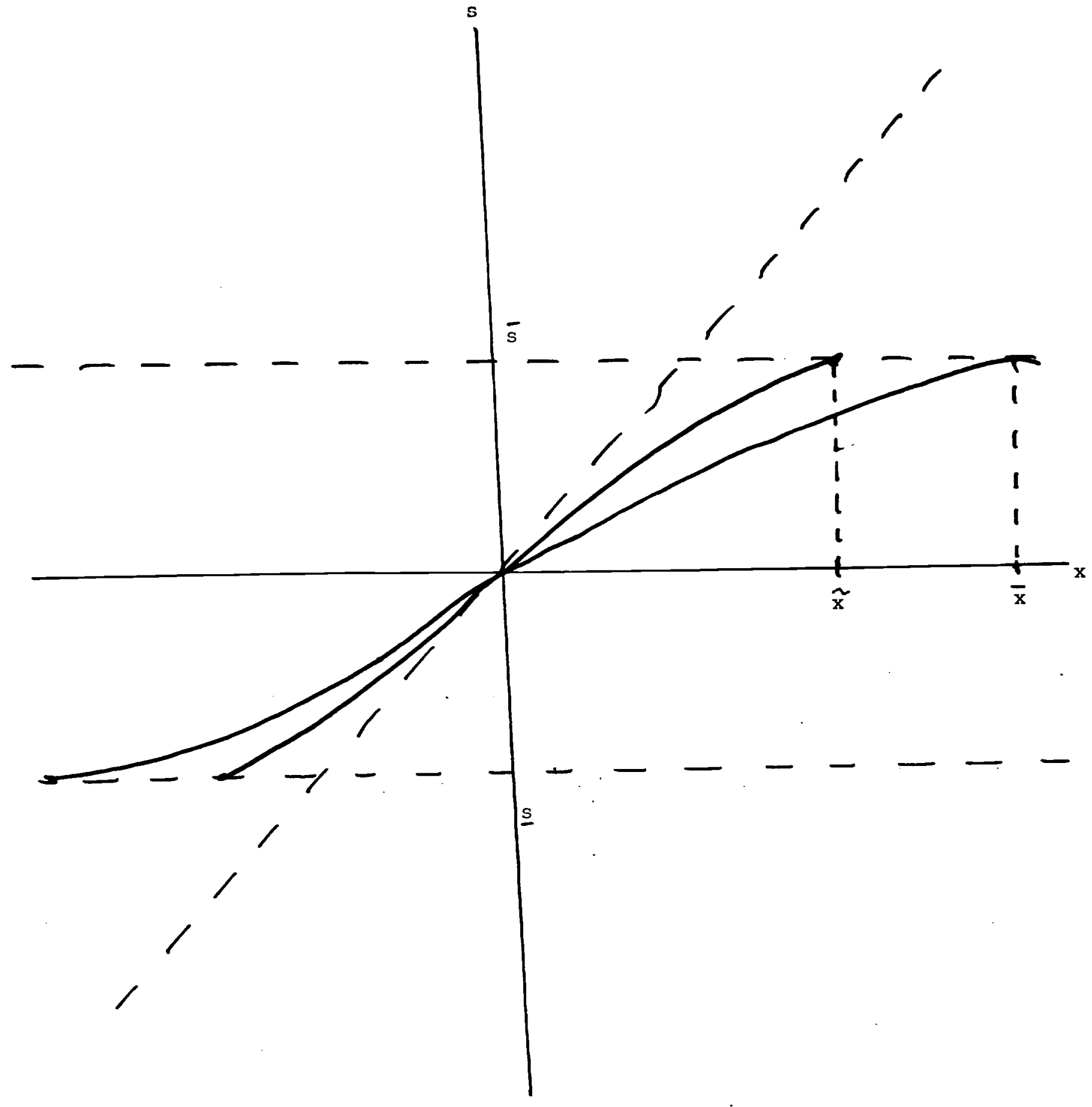

Figure 3: Effects of imperfect credibility 